\title{
Adapting Psychosocial Intervention Research to Urban Primary Care Environments: A Case Example
}

Luis H. Zayas, $P b D^{1}$

M. Diane McKee, $M D, M S^{2}$

Katherine R. B. Jankowski, $M A^{3}$

'Center for Mental Health Services Research, George Warren Brown School of Social Work, Washington University in St. Louis, St. Louis, Mo

${ }^{2}$ Albert Einstein College of Medicine, Bronx, NY

${ }^{3}$ Department of Psychology, Fordham University, Bronx, NY

Conflicts of interest: none reported

\section{CORRESPONDING AUTHOR}

Luis $\mathrm{H}$. Zayas, $\mathrm{PhD}$

Center for Mental Health Services Research George Warren Brown School of Social Work Washington University in St. Louis One Brookings Drive, Campus Box 1196 St. Louis, MO 63130-4899

lzayas@wustl.edu

\begin{abstract}
PURPOSE We wanted to describe the unique issues encountered by our research team in testing an intervention to reduce perinatal depression in real-world community health centers.

METHOD We used a case study of an experience in conducting a randomized controlled trial designed to test the effectiveness of a low-cost multimodal psychosocial intervention to reduce prenatal and postpartum depression. Low-income minority women $(\mathrm{N}=187)$ with low-risk pregnancies were randomly assigned to the intervention or treatment as usual. Outcomes of interest were depressive symptoms and social support assessed at 3 months' postpartum.

RESULTS Our intervention was not associated with changes in depressive symptoms or social support. Challenges in implementation were related to participant retention and intervention delivery. Turnover of student therapists affected continuity in participant-therapist relationships and created missed opportunities to deliver the intervention. The academic-community partnership that was formed also required more involvement of health center personnel to facilitate ownership at the site level, especially for fidelity monitoring. While attentive to cultural sensitivity, the project called for more collaboration with participants to define common goals and outcomes. Participatory research strategies could have anticipated barriers to uptake of the intervention and achieved a better match between outcomes desired by researchers and those of participants.
\end{abstract}

CONCLUSION Several criteria for future research planning emerged: assessing what the population is willing and able to accept, considering what treatment providers can be expected to implement, assessing the setting's capacity to accommodate intervention research, and collecting and using emerging unanticipated data.

Ann Fam Med 2004;2:504-508. DOI: 10.1370/afm.108.

\section{INTRODUCTION}

$\mathrm{D}$ uring the past 3 decades, primary care practice-based research has made notable progress in establishing its feasibility and potential to address questions important to primary care and the communities we serve. ${ }^{1-5}$ Practice-based research can answer questions of greater relevance to primary care practice, test effectiveness of treatments in undifferentiated patient populations, ${ }^{3}$ and engage clinicians in the generation of new knowledge that can be readily assimilated into practice. ${ }^{5}$ Despite gains made, researchers note that challenges persist in successful implementation of studies in primary care settings. ${ }^{6-8}$ Moving psychosocial intervention research into urban community-based primary care settings that are not research oriented remains highly challenging. Disappointing results of well-designed intervention research may be due to practical difficulties of implementing studies in unpredictable community-based health centers rather than to lack of intervention efficacy ${ }^{9-11}$ Barriers, expected and unex- 
pected, to implementing intervention research in community-based settings will remain ${ }^{1,2,12}$ and will likely be exacerbated in the unique setting of safety net sites. ${ }^{13}$

In this article, we describe logistical hurdles encountered in testing an intervention to reduce depression in pregnant, low-income urban minority women in community health centers. We describe the varied elements that affected our research: the persons who receive interventions, the persons who provide interventions, and the persons who collectively create service delivery systems - contexts subject to the effects of unanticipated events. We offer several criteria for adapting intervention research to real-world circumstances.

\section{METHODS}

Participants were 187 African American and Hispanic women with low-risk pregnancies (mean age $=$ 25 years; mean education $=12$ years). Women were screened for depressive symptoms in the third trimester, and those with elevated depressive symptoms were randomly assigned to an intervention group $(\mathrm{n}=57)$ or group receiving treatment as usual $(n=43)$ offered by their health center. A third group of women who were not depressed $(n=87)$ was used for comparison. Data were collected again at 2 weeks' and 3 months' postpartum. ${ }^{14,15}$ Depression was measured with the Beck Depression Inventory, second edition (BDI-II ), in which a score of 14 is considered the lower end of depressive symptoms. ${ }^{16}$ Total functional social support (ie, actual received support) was measured with the Norbeck Social Support Questionnaire, an instrument for use with pregnant women. ${ }^{17}$ Life stressors were measured with the Life Events Questionnaire, ${ }^{18}$ and functional abilities were measured with the Medical Outcome Study Short Form-36 (SF-36). ${ }^{19}$

The intervention consisted of 3 components: an 8 -session cognitive behavioral treatment specifically developed for depression prevention in primary care, ${ }^{20}$ 4 psychoeducation sessions using videotaped and written materials on infant development and maternal sensitivity, and ongoing social support building from the therapist. Therapists were graduate students in social work (all women of an ethnic minority completing clinical internships) who were trained and supervised in both the intervention and cultural sensitivity. Intervention sessions were offered in the health center or at home at least twice a month.

The settings were 3 health centers in the south Bronx, NY, all of which had prenatal teams of physicians, nurses, and social workers. The 2 larger, federally funded community health centers had formal psychosocial service teams. The smallest site was a family practice setting with a part-time social worker. The sites had little experience with research and none with intervention studies.

\section{RESULTS}

We describe the challenges and lessons learned in implementing and measuring study effects. Thirtyeight percent of the sample was lost to attrition. Of this $38 \%, 42 \%$ and $44 \%$ of those in the intervention and treatment-as-usual groups, respectively, did not complete the study (Table 1 ). Only $32 \%$ of the women who were not depressed dropped out of the study. No significant differences were apparent in changes in total functional social support or reduction of depression in the 2 treatment groups.

Methodological and logistical challenges arose that affected outcomes, some from the realities of the research contexts and some through errors of commission and omission.

\section{Community Health Center Structures}

The reputations of the health centers aided patient recruitment, but conducting an intervention in 3 health centers required negotiating with different systems and staff attitudes toward research. Administrators feared that service productivity and the bottom line would be compromised rather than enhanced by an intervention study. Occasional chaos broke out in health centers from operational changes wrought by regulatory and funding authorities. ${ }^{21}$ Collaborating with different sets of clinicians facing extraordinary demands from patients and administration also affected delivery of the intervention. Other interruptions occurred when equipment or space was unavailable, requiring sudden adjustments in plans for sessions and many missed treatment opportunities.

\section{Therapists and Intervention Fidelity}

Constraints of the academic calendar on student-therapists affected the continuity of therapeutic relationships, leading us to conclude that engaging full-time on-site psychosocial providers might have resulted in both more sessions delivered and less attrition. Concurrently, the limited ability to monitor the fidelity of the intervention owing to space constraints, lack of secure storage for recording equipment, and logistical barriers to videotaping or audio recording the intervention sessions affected implementation. Although we were successful in an important goal of minimizing the intrusiveness of our study to the practices, greater involvement of center personnel in problem solving, especially the presence of an on-site champion, might have encouraged a greater sense of ownership in the project and commitment to facilitating fidelity monitoring. ${ }^{6-8}$ 
Patient Adherence to the Intervention

Although participants were well informed of what the study entailed, through their behavior and comments it was apparent that they determined-generally by default - what parts of the planned intervention they would or could accept. Often, these choices were dictated by life circumstances (eg, caring for a newborn, a sick child, financial problems, competing appointments) rather than simple refusals. We found less acceptance of home visits than expected; reasons cited included the social activities of health centers, concerns for the safety of our therapists, and occasionally the opposition of partners. Adherence might have been enhanced with a more systematic collection and analysis of qualitative information so we could adapt our protocol to the women's real-life circumstances. Strategies from participatory research could have anticipated these barriers by collaboration with members of our target community throughout study development and implementation. ${ }^{21,22}$

\section{Relevance of Outcomes}

We encountered differences in what researchers consider relevant patient outcomes and what patients think are relevant outcomes for themselves. ${ }^{10}$ Women described themselves as stressed, not depressed; thus, those elements of the intervention aimed toward reducing depressive symptoms may have seemed irrelevant to many women. We learned that getting social support or psychoeducational information, having a compassionate listener, or learning about available childcare was more important to them than reducing depression or engaging in pleasurable activities. Participatory research strategies that included patients' and other stakeholders' voices in all stages of the project, even if it involved modifying the goals initially set for the study, would have created a better match in the preferred outcomes of patients and researchers. ${ }^{21,22}$

\section{Retention of Participants}

As Table 1 shows, retaining participants for a 4 - to 6 month period was a major challenge. This issue is especially common in studies of urban, minority poor popu- lations. ${ }^{23,24}$ The turnover in student therapists affected some participants' willingness to be transferred to new therapists. Motivation to continue may have waned for some clients as a result of a mismatch of the intervention components to women's perceived needs.

\section{Uptake of the Intervention}

Providing full dosages of a varied intervention proved to be more than what the women's lives allowed, even with therapists' best efforts and with the most motivated participants. The intervention group received an average of 3 of 8 planned cognitive behavioral treatment sessions (range 0 to 7 ), 1 of 4 planned child development sessions (range 0 to 3 ), and 2 of an indefinite number of social-support-building sessions (range 0 to 14 ), with substantial variability for individual women. We found that women who reported more positive life events and more social support and better perceived general health availed themselves of more treatment than those with less social support, low perceived health, and fewer positive life events, suggesting that delivering interventions may be most problematic for those with greatest need.

\section{CONCLUSION}

We concur with others that the adaptations and findings of field trials may actually be stronger than those of laboratory studies because of the more demanding, fluid research environments in which they are conducted. ${ }^{25}$ Thus, practice-based research has greater relevance and applicability to the primary care patients we serve. ${ }^{2,3}$ We affirm the reality that intervention research is difficult to implement faithfully in urban primary care settings, especially those without a tradition or infrastructure for research. Our project represented an academic-community partnership, but we had insufficient involvement of the community practices to facilitate ownership at the site level or optimal problem solving, especially beyond its initial months. While we were attentive to cultural sensitivity, we lacked the true collaboration with participants necessary to define common goals and outcomes. ${ }^{6,22}$

Table 1. Summary of Attrition and Depression Scores

\begin{tabular}{lcrrrrr}
\hline & \multicolumn{3}{c}{ Third Trimester } & \multicolumn{2}{c}{ 3 Months' Postpartum } \\
\cline { 2 - 6 } Groups & No. (\%) & BDI-II (SD) & $\begin{array}{c}\text { Total Functional } \\
\text { Support(SD) }\end{array}$ & No. (\%) & BDI-II (SD) & $\begin{array}{c}\text { Total Functional } \\
\text { Support (SD) }\end{array}$ \\
\hline Intervention & $57(100)$ & $20.5(6.2)$ & $97.4(68.5)$ & $32(58.1)$ & $12.1(6.4)$ & $84.3(44.1)$ \\
Treatment as usual & $44(100)$ & $22.4(7.0)$ & $75.7(46.0)$ & $25(56.1)$ & $12.6(7.0)$ & $5.9(5.3)$ \\
Nondepressed comparison & $86(100)$ & $8.4(3.0)$ & $101.1(51.3)$ & $59(67.8)$ & $93.9(46.6)$ \\
\hline BDI-II = Beck Depression Inventory, 2nd edition, score.
\end{tabular}


Our key message is that psychosocial intervention research in community-based primary care will require flexibility in adjusting the research design before and during implementation..$^{10}$ The concerns of multiple stakeholders, nonresearch settings, and real-life issues in patients' lives can be reconciled by research designs that result in cycles of outcome assessments and procedure refinements. ${ }^{26}$

Observing several criteria may help adapt research design to stakeholder and service system characteristics:

1. Assess what the target population can and will accept of an intervention. Using participatory research approaches, patients and knowledgeable staff can answer such questions as, What are the key issues in patients' lives that are essential to target with the intervention? What kinds of interventions and in what dosages will patients accept? What kinds of outcomes do patients want and in what domains? Which outcomes will be most compelling to patients? Answers to these questions are contingent on understanding service and community contexts early on in a project. Operationally, this understanding may be accomplished by partnering with community representatives and conducting key stakeholder focus groups and interviews.

2. Aim toward interventions that clinicians in comparable settings can deliver, given the typical demands of practice in community settings. This criterion would pose such questions as, What will clinicians in community settings like this one need to know? What intervention and how much of the intervention can clinicians reasonably be expected to deliver? What will they embrace and what will they reject? Clinicians' acceptance of empirically-based interventions in these circumstances is more likely to occur when combinations of treatments with small doses are available and where training requirements are not excessive. Despite the demands of the primary care setting, training onsite personnel to deliver the intervention might raise their ownership of the study and help identify potential problems and realistic solutions.

3. Assess the service setting's capacity to accommodate intervention research. How does the intervention researcher integrate externally and internally induced changes in the service system into the research protocol? Countless predictable and unpredictable changes in participating practices can disrupt implementation and data collection. In every dimension of research, adaptations might be required that deviate from the original plans; these adaptations can be creatively pursued while maintaining the fundamental research design. This criterion obviates the need for ongoing discussions with providers and administrators in preparing for impending changes, particularly in nonresearch environments. Looking ahead at looming policy or fis- cal realities that will bring changes to service systems and asking how the centers managed similar upheavals in the past can prepare the research project staff to reduce interruptions and adapt in advance.

4. Collect emerging data systematically that are unanticipated or not easily quantifiable but which may be important later. Typically, this information is not originally considered as part of the data to be collected. Yet the events that occur can have powerful effects on research implementation. The question then becomes, How can emerging, even anecdotal information, be collected and reviewed systematically to make appropriate design adaptations while maintaining scientific integrity? We recommend encouraging regular input of anecdotes and hunches from all members of the team, recording and examining these systematically for indications of trends and troubles. Our experience shows that unexpected findings or implementation problems can lead to creative and useful research adaptations.

To read or post commentaries in response to this article, see it online at http://www.annfammed.org/cgi/content/full/2/5/504.

Key words: Psychosocial treatment; intervention research, primary health care; perinatal depression; mental health

Submitted March 3, 2003; submitted, revised, October 12; 2003; accepted October 182003.

Funding support: Financial support was provided, in part, by National Institute of Mental Health grants R24 MH57936 and R24 MH60002.

\section{References}

1. Nutting PE, Beasley JW, Werner JJ. Practice-based research networks answer primary care questions. JAMA. 1999;281:686-688.

2. Stange K. Primary care research: barriers and opportunities. J Fam Pract. 1996;42:192-198.

3. Green LA, Hames CG, Nutting P. Potential of practice-based research networks: experiences from ASPN. J Fam Pract. 1994;38:400-406.

4. Yawn B. Conference Proceedings. Methods for Practice-Based Research Networks: Challenges And Opportunities. Leawood, Kan: American Academy of Family Physicians; 2001:13-14.

5. Stange K, Miller W, McWhinney I. Developing the knowledge base of family practice. Fam Med. 2001;33:286-297.

6. Christoffel KK, Binns HJ, Stockman JA, et al, and the Pediatric Practice Research Group. Practice-based research: opportunities and obstacles. Pediatrics. 1988;82:399-406.

7. Beasley JW. Success and failure in practice-based research: WreN. In: Practice-Based Research Networks in the 21st Century, Conference Proceedings. Leawood, Kan: American Academy of Family Physicians; 1998:65-71.

8. Hickner J. The best and worse studies of the Upper Peninsula Research Network. In: Practice-Based Research Networks in the 21st Century, Conference Proceedings. Leawood, Kan: American Academy of Family Physicians; 1998;72-75.

9. Farmer EMZ, Burns BJ, Guiles HB, Behar L, Gerber D. Conducting randomized clinical trials in children's mental health: experiences and lesson from one venture. In: Nixon C, Northrup D, eds. Evaluating Mental Health Services: How Do Programs for Children "Work" in the Real World? Thousand Oaks, Calif: Sage Publications; 1997. 
10. Hohman AA, Shear MK. Community-based intervention research: coping with the noise" of real life in study design. Am J Psychiat. 2002; 159:201-207

11. Rogers S, Humphrey C, Nazareth I, Lister S, Tomlin Z, Haines A. Designing trials of interventions to change professional practice in primary care: lessons from an exploratory study of two change strategies. BMJ. 2000;320:1580-1583.

12. Klinkman MS, Okkes I. Mental health problems in primary care: a research agenda. J Fam Pract. 1998;47:379-384

13. Dietrich AJ, Tobin JN, Sox $\mathrm{CH}$, et al. Cancer early-detection services in community health centers for the underserved. A randomized controlled trial. Arch Fam Med. 1998;7:320-327.

14. McKee MD, Cunningham M, Jankowski KRB, Zayas LH. Healthrelated functional status in pregnancy: relationship to depression and social support in a multi-ethnic population. Obstet Gynecol. 2001;97:988-993.

15. Zayas LH, Cunningham M, McKee MD, Jankowski KRB. Depression and negative life events among pregnant African-American and Hispanic women. Women's Health Issues 2002;12:16-22.

16. Beck AT, Steer RA, Brown GK: BDI-II: Beck Depression Inventory Manual. 2nd ed. San Antonio, Tex: Psychological Corporation; 1996.

17. Norbeck JS, Lindsey AM, Carrieri VL. Further development of the Norbeck Social Support Questionnaire: Normative data and validity testing. Nursing Res. 1983;32:4.
18. Norbeck JS, Anderson NJ. Life stress, social support, and anxiety in mid- and late-pregnancy among low income women. Res Nursing Health. 1989;12:281-289.

19. Ware JE. Short Form-36 Health Survey: Manual and Interpretation Guide. Boston, Mass: The Health Institute, New England Medical Center; 1993.

20. Miranda J, Muñoz RF. Intervention for minor depression in primary care patients. Psychosomatic Med. 1995;56:136-142.

21. Green LW, Mercer SL. Can public health researchers and agencies reconcile the push from funding bodies and the pull from communities? Am J Public Health. 2001;91:1926-1929.

22. Macaulay AC, Commanda LE, Freeman WL, et al. Participatory research maximizes community and lay involvement. BMJ. 1999;319:774-778.

23. Miranda J, Azocar F, Organista KC, Muñoz RF, Lieberman A. Recruiting and retaining low-income Latinos in psychotherapy research. J Consult Clin Psychol. 1996;64:868-874.

24. Thompson EE, Neighbors HW, Munday C, Jackson JS. Recruitment and retentions of African American patients for clinical research: an exploration of response rates in an urban psychiatric hospital. J Consult Clin Psychol. 1996;64:861-867.

25. Hoagwood K., Hibbs T, Brent D, Jensen P. Introduction to the special section: efficacy and effectiveness in studies of child and adolescent psychotherapy. J Consult Clin Psychol. 1995;63:683-687.

26. Weisz JR, Donenberg GR, Han SS, Weiss B. Bridging the gap between laboratory and clinic in child and adolescent psychotherapy. J Consult Clin Psychol. 1995;63:688-701. 